\title{
CURRENT TRENDS IN HOME HEALTH CARE
}

\author{
Suguna Mary.D, Rajalakshmi.R*,
}

\begin{abstract}
India has the second largest geriatric population in the world. Of the 300 million over-60s, 200 million are likely to be suffering from chronic ailments, and so it is essential to concentrate on Home healthcare includes supportive, preventive, promotive and rehabilitative services with the facilities available at the patient's home. Home health care helps them get better, regain independence, and become as self-sufficient as possible. It also helps to ensure a "good quality of life." This article focuses on the Current Trends in Home health Care and the Standards, Challenges and the future plans in Home health Care nursing..
\end{abstract}

Key words: Home health care, Caregiver, Agencies, Nursing Standards छ Practice.

\section{Introduction}

India has the second largest geriatric population in the world. Of the 300 million over-60s, 200 million are likely to be suffering from chronic ailments. Already, non-communicable diseases including cardiovascular diseases, diabetes, chronic obstructive pulmonary disease and cancer cause around 50\% of all deaths in India, and so it is essential to concentrate on Home healthcare that includes supportive, preventive, promotive and rehabilitative services with the facilities available at the patient's home. Devices needed for patient are provided at home itself. These care services include Respiratory Therapy, Home Infusion Therapy, Rehabilitation Services, and Palliative Care. The most significant healthcare trend witnessed recently is the shift of treatment from hospitals to home in order to gain a cost advantage and reduce hospital expenditure. The move from treatment to proactive monitoring is also opening up new opportunities for this market. Patients prefer home healthcare over hospitals mainly for the convenience and cost-effectiveness it offers. ${ }^{[1]}$

"Home care", "home health care" and "In-home care" are phrases that are used interchangeably to mean any type of care given to a person in their own home. These phrases have been used in the past interchangeably regardless of whether the person required skilled care or not. More recently, there is a growing movement to distinguish between "home health care" meaning skilled nursing care and "home care" meaning non-medical care.

Home care aims to make it possible for people to remain at home rather than use residential, longterm, or institutional-based nursing care. Home care providers deliver services in the client's own home. These services may include some combination of professional health care services and life assistance services. Professional home health services could include medical or psychological assessment, wound care, medication teaching, pain management, disease education and management, physical therapy, speech therapy, or occupational therapy. Home care is often an integral component of the post-hospitalization recovery process, especially during the initial weeks after discharge when the patient still requires some level of regular physical assistance. ${ }^{[1]}$

\section{Definition}

Home health care refers to all of the services $\&$ products provided to clients in their homes to maintain, restore, or promote their physical, mental, 
\& emotional health. Its purpose is to maximize the clients level of independence \& to minimize the effects of existing disabilities through non institutional services. ${ }^{[15]}$

\section{The Standards of home health care are:}

We're leaving the hospital earlier and thus need more intensive care.

Sophisticated medical technology has moved into our homes. Devices are now in our living rooms and bedrooms. ${ }^{[3]}$

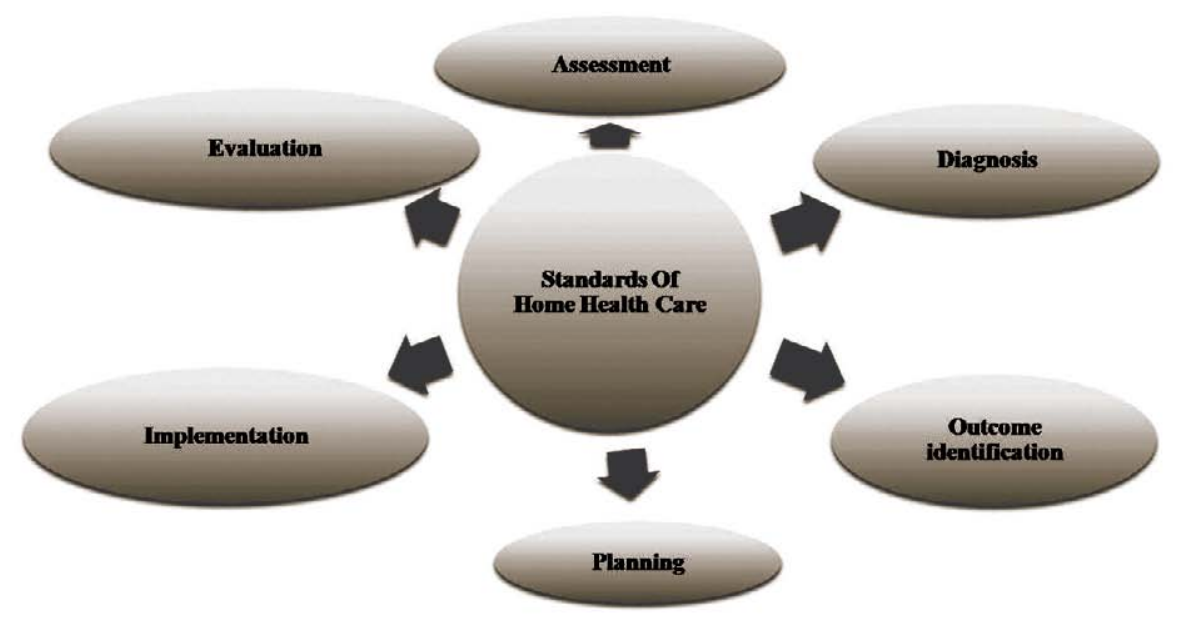

\section{Aspects of Home Health Care}

Most of the home care is informal, with families and friends providing a substantial amount of care. For formal care, the health care professionals most often involved are nurses followed by physical therapists and home care aides. Other health care providers include respiratory and occupational therapists, medical social workers and mental health workers. Home health care is generally paid for by Medicaid, Medicare, long term insurance, or paid with the patient's own resources. The goal of home health care is to treat an illness or injury. Home health care helps you get better, regain your independence, and become as self-sufficient as possible. It also helps to ensure a "good quality of life."A number of interacting factors directly affect home health care the capabilities of patients and caregivers, the tasks and medical therapies undertaken, the devices and technologies used, and the physical as well as community environment in which all this occurs. ${ }^{[2]}$

\section{Need for Home Health care}

We're living longer and more of us want to "age in place with dignity."

We have more chronic, complex conditions.

\section{Standards of Professional Practice:}

- Quality of care: systematically evaluates the quality \& effectiveness of nursing practice.

- Performance appraisal: evaluates the nursing practice standards, scientific evidences \& regulations.

- Education: should update and maintain current knowledge and competency in nursing practice.

- Collegiality: contributes to the professional development of peers \& other health care providers as colleagues.

- Ethics: home health nurse's decisions \& actions on behalf of clients are determined in an ethical manner.

- Collaboration: essential in providing home health care.

- Research: Home health Nurse uses research findings in practice.

- Resource utilisation: Home Health Nurse assists the client or family in becoming informed consumers about the risks, benefits $\&$ cost of planning \& delivering client care. ${ }^{[14]}$

\section{Types of Home Care Personnel}

Most people receiving home care receive care from to or more caregivers, either formal or informal. 


\section{Informal Caregivers:}

Are family members \& friends who provide care in the home. They provide routine custodial care such as bathing \& feeding to sophisticated skilled care, including tracheostomy care, IV medication administration.

\section{Formal caregivers:}

Are professionals \& paraprofessionals who are compensated for the In - home care they provide.

\section{Types of Home Care Agencies:}

\section{Voluntary agencies:}

Financed with non tax funds such as donations, endowments and third party provider payments.

\section{Proprietary agencies:}

Are expected to turn a profit on the services they provide, either for the individual owners or stock holders.

\section{Hospital based agencies:}

Governed by the sponsoring hospital board of directors or trustees.

\section{Official Agencies:}

Created and empowered through statutes enacted by legislations. Services are frequently provided by the nursing divisions of state or local health departments.

\section{Hospices:}

Promotes a care perspective that recognizes that death is inevitable and cure is not at present a possibility. Services are comprehensive and are delivered by an interprofessional team, including volunteers, that focuses on"Care" rather than "Cure". [6] [12] [15]

\section{Trends in home health care:}

\section{Chronic Care, Everywhere:}

Home healthcare can provide management of chronic illnesses more cost effectively than hospitals or nursing homes. Since each chronic condition increases costs by a factor of three, managing this population is the sweet spot for the ACO (accountable care organizations), and the deepest pool from which to pull savings. To do it, an increasing number of providers will deploy Ambulatory Intensive Care Units (A-ICUs) or patient centered medical homes as part of their ACO, which will be charged with better managing chronic conditions exclusively within a clinically integrated, financially accountable primary care practice. As part of the approach, providers will develop care pathways for better managing chronic conditions and behavioral health needs, with an eye toward lowering hospital utilization, including inpatient bed days, length of stay, admissions, readmissions, and emergency department visits. ${ }^{[14]}$

\section{Hospital at Home.}

Also driving the growth is the need to make care more convenient, particularly for those with chronic conditions, so patients can be monitored and coached to health anytime, anywhere. And there's a cost component to the trend as well. "Hospital at Home," a program designed by Johns Hopkins that provides acute care services in the homes of patients who might otherwise be hospitalized, has been demonstrated to increase the quality of care patients receive, improve their satisfaction, and reduce costs by at least 30 percent. ${ }^{[1]}$

\section{Virtual Care at Home}

Technology-enabled at-home health care is increasingly solving an access issue for patients. According to a recent survey, almost half of rural hospitals use virtual care or telemedicine to connect with patients who may be too far away for an inperson visit, allowing them to close the gaps in care that arise due to geography. ${ }^{[2]}$

\section{Rehabilitation Nurses}

Rehabilitation nursing is a specialty practice that is committed to improve the quality of life for individuals with a disability or a chronic illness. The rehabilitation nurses mission is to improve the optimal level of functioning of individuals with a disability or a chronic illness at home and in the community. The goal of the rehabilitation process is to provide, in collaboration with an interdisciplinary healthcare team that includes the client, a holistic approach to nursing care that maximizes the clients independence and mastery of self-care activities. 
Physical, emotional, social, cultural, educational, developmental, and spiritual dimensions are considered when team members establish goals for the client. ${ }^{[7][9]}$

\section{Examples of skilled home health services}

$>$ Wound care for pressure sores or a surgical wound

$>$ Patient and caregiver education

$>$ Intravenous or nutrition therapy

$>$ InjectionsMonitoring serious illness and unstable health status ${ }^{[12]}$

\section{Roles of the home health nurse}

\section{Practitioner}

- Acts as a resourceful caregiver during a crisis that is aggravated by a chronic illness or a disabling condition.

- Implements nursing care based on scientific knowledge, home care standards, and principles that are appropriate to the home care environment

- Collaborates with the interdisciplinary team in the management of the team function in the home environment; is responsible for ensuring that the client is involved as a significant member of the team.

- Helps the client and the client's family adapt to changes in lifestyle necessitated by the disabling condition.

- Assesses the appropriateness of a client's admission to, and the delivery of rehabilitation services in, the home environment. ${ }^{[13]}$

\section{Care coordinator}

- Acts as a member of the interdisciplinary healthcare team and promotes the coordination of client care, the activities of other professionals. Integrates the knowledge and skills of various professional and non-professional disciplines into a comprehensive continuum of care \& Facilitates the design and implementation of the plan of care for clients who are chronically ill or who have disabling conditions. ${ }^{[5]}$

\section{Advocate}

- Advocates for clients and their families or caregivers \& Teaches clients and their families or caregivers to advocate for themselves.
- Facilitates the client's transition from the hospital to the home and the community.

- Furthers an understanding of home care-based rehabilitation issues among people in the community and among those in government who are in a position to deal with issues related to this patient population.

\section{Educator}

- Provides education for clients and their families

- Provides staff orientation and guides staff development, both at the professional and the paraprofessional levels, in the area of rehabilitation home care \& Provides rehabilitation-focused continuing education programs.

- Develops policies and procedures that are specific to rehabilitation home care \& Develops educational materials designed to help clients and their family members become knowledgeable consumers in the healthcare arena. ${ }^{[10]}$

\section{Consultant}

- Identifies clients and families who could benefit from home care services.

- Provides case management expertise within the home care environment.

- Serves as a liaison with third-party payers and justifies the use of funds for home care.

- Promotes nursing services to community health professionals and to the community at large.

\section{Researcher}

- Participates in research involving home care clients and their families.

- Participates in the analysis and dissemination of evaluative data that may have an impact on clients and their families.

- Incorporates evaluative data into nursing practice. ${ }^{[8]}$

\section{Challenging Factors In Home Health \\ Care}

* Increased demands.

- Care providers: licensed nurses, certified care providers

- Client care tracking and service recording

* Long-term healthcare coverage

- Fixed-rate increase by Medicare and Medicaid 
Types of home care: respite, long-term, hospice, and elderly

* Supplies: equipment, transportation, medical, and special needs

* Rise in travel costs for care providers

* Home healthcare agency franchises (increase in profits)

* Seniors choosing to live at home and receive care

* Hospice care training for care providers. ${ }^{[7][4]}$

Working as a Home Care Nurse has drawbacks, such as having fewer opportunities to consult with colleagues about challenges than in other settings such as a hospital. The median annual salary of Home Care Nurse is below the median hospital nurse's salary. The rewards of being a Home Care Nurse far outweigh any disadvantages. Having one-to-one client interaction fosters an authentic and personal nurse/client relationship. Those $\mathrm{CHNs}$ working with community groups can affect the health of many and have input about how scarce resources are used. On the practical side, Home Care Nurse have a more flexible work schedule than hospital-based nurses and more independence. Home Care Nurse is challenging, rewarding, and filled with professional and personal satisfaction. ${ }^{[1]}$

\section{Building for Tomorrow}

With life expectancy increasing significantly in India, home health care is becoming mandatory. As a result of advances in communication and medical technology, a lot of interventions that could earlier take place only in hospital settings are now becoming possible in the home environment. Specialty home health care will be a key element of patient-centric care in the coming years. The task of understanding the complexities of the home health care environment so that high quality and safe care can occur." As the demand increases for home healthcare a care tracking solution is needed. "The Indian educational system currently does not have a structured training program for home health care. Inorder to render specialised, evidence based care a clear, evidence-based treatment protocols that cover the range of protocols should be delivered by the experts team. Government has to offer some special courses for the Community Health Nurses to overcome the bottleneck factors so that a personalised care will be rendered by a qualified Home Health Care Provider. ${ }^{[7]}$

\section{References}

1. "Hospital to Homecare". Hospital to Homecare. Retrieved 10 March 2012.

2. Emerging Trends: Indian Healthcare Industry December-2013 Hospital Services Industry: Riding on the back of growing healthcare demand; available at; http:// www.onicra.com/images/pdf/Healthcare-industry-reportTransparent.pdf

3. Human factors challenges in home health care: Research Activities, December 2011, No. 376. December 2011.

4. Christensen, L.R.; E. Grönvall (2011). "Challenges and Opportunities for Collaborative Technologies for Home Care Work". Aarhus, Denmark (Springer): 61-80. DOI :10.1007/978-0-85729-913-0 4. Retrieved 24 July 2013.

5. Patrick A. Cunningham, Home Healthcare Nurse: The Role of the Psychiatric Nurse in Home CareNovember/December 2007 - Volume 25 - Issue 10 - p 645-652 DOI: 10.1097/01. NHH.0000298935.76211.07

6. "Home Health services" - BAYADA Home Health Care retrieved 2013-07-19

7. Susan D, The Changing Health Care World: Trends To Watch In 2014.DOI:feb,10 2014; available at: http://healthaffairs. $\mathrm{org} / \mathrm{b} \log / 2014 / 02 / 10 /$ the-changing-health-care-worldtrends-to-watch-in-2014/

8. Rising Trends In Health Care: Body and Mind staff ; Avilable at http://www.pennlive.com/bodyandmind/index. ssf/2011/02/5_rising_trends_in_health_care.html: 2011.

9. The Home Care Rehabilitation Nurse: association of rehabilitation nurses;DOI:feb,02 2015, available at: http:// www.rehabnurse.org/pubs/role/Role-The-Home-CareRehab-Nurse.html

10. Kulbok, P.A., Thatcher, E., Park, E., Meszaros, P.S. (May 31, 2012) "Evolving Public Health Nursing Roles: Focus on Community Participatory Health Promotion and Prevention" OJIN: The Online Journal of Issues in Nursing Vol. 17, No. 2, Manuscript 1.DOI: 10.3912/OJIN. Vol17No02Man01

11. Ricco,Patricia.A (2001) “ Quality Evaluation of Home Nursing Care: Perceptions of Patients, Physicians and Nurses". Journal of Nursing Care Quality 15 (2):58 - 67. DOI: 27 July 2008.

12. Lotus Shyu, Yea-Ing; Hsiao-Chin Lee (2002). "Predictors of nursing home placement and home nursing services utilization by elderly patients after hospital discharge in Taiwan". Journal of Advanced Nursing 38(4): 398-406. DOI:10.1046/j.13652648.2002.02193.x.PMID 1198561.

13. Modin, S.; A. K. Furhoff (2002). "Care by general practitioners and district nurses of patients receiving home nursing: a study from suburban. Scandinavian Journal of Primary Health Care 20 (4): 208-212(5). DOI:10. 1080/ 0281343023210048 54. Retrieved 27 July 2008.

14. Scope and Standards of Home healthnursing practice. Washington, D C; American Nurses Publishing, American NursesAssociation (1999)

15. Judithann Allender, Promoting andProtecting the Public's Health Community Health Nursing,6th edition, Lippincott Williams \& Wilkins, pg: 886 - 890 . 